\title{
A NOTE ON ORLICZ ALGEBRAS
}

\section{Seyyed Mohammad Tabatabaie, Ali Reza Bagheri Salec and MARYAM ZARE SANJARI}

Abstract. The purpose of this paper is to give a necessary and sufficient condition for an Orlicz space $L^{\Phi}(G)$ to be a convolution Banach algebra, where $G$ is a compactly generated locally compact abelian group and $\Phi$ is a Young function satisfying $\Delta_{2}$-condition and an extra sequence condition.

Mathematics subject classification (2010): 46E30, 43A15.

Keywords and phrases: Locally compact group, Orlicz algebra, convolution, Young function, N-function, Zygmund space.

\section{REFERENCES}

[1] F. Abtahi, R. NASR Isfahani And A. ReJali, On the $L_{p}$-conjecture for locally compact groups, Arch. Math. (Basel) 89, (2007), 237-242.

[2] C. Chen AND C.-H. Chu, Hypercyclic weighted translations on groups, Proc. Amer. Math. Soc. 139, (2011), 2839-2846.

[3] E. Hewitt And K. A. Ross, Abstract Harmonic Analysis, Springer-Verlag, Heidelberg, 1979.

[4] H. HudziK, A. KAmiska AND J. Musielak, On some Banach algebras given by a modular, in: Alfred Haar Memorial Conference, Budapest, Colloquia Mathematica Societatis J anos Bolyai (North Holland, Amsterdam), 49, (1987) 445-463.

[5] M. M. RaO AND Z. D. Ren, Theory of Orlicz Spaces, Marcel Dekker, New York, 1991.

[6] A. OSANÇLIOL AND S. ÖZTOP, Weighted Orlicz algebras on locally compact groups, J. Aust. Math. Soc. 99, (2015), 399-414.

[7] K. URBANiK, A proof of a theorem of Zelazko on $L^{p}$-algebras, Colloq. Math. 8, (1961), 121-123.

[8] W. ZelazKo, A note on $L^{p}$-algebras, Colloq. Math. 10, (1963), 53-56. 